\title{
Alterations in Cardiac Vagal Modulation-to-Vagal Tone Ratio in response to accumulated exercise stress in intermittent team sport
}

\author{
Andrew S. Perrotta ${ }^{1,2}$, Darren E.R. Warburton ${ }^{2,3}$ \\ 1 Department of Kinesiology, Langara College, Vancouver, Canada; ${ }^{2}$ Faculty of Medicine, Experimental Medicine \\ Program, University of British Columbia, Vancouver, Canada; ${ }^{3}$ School of Kinesiology, Faculty of Education, University of \\ British Columbia, Vancouver, Canada
}

\section{Summary}

Study aim: This investigation examined the cardiac vagal modulation-to-vagal tone ratio and its utility to represent the dose response to accumulated exercise stress in intermittent team sport.

Material and methods: Fourteen international calibre female field hockey players training as part of a national team were participants over a three-week period. Exercise stress was expressed through cardiovascular strain using heart rate (HR) dynamics and was quantified as a training load (AU) and time spent exercising above anaerobic threshold (min). Cardiac autonomic activity was examined using heart rate variability; R-R intervals (ms) were collected immediately upon awakening throughout each weekend. The square root of the mean squared differences of successive R-R intervals were individually calculated, and $\log$ transformed prior to being averaged (Ln rMSSD) along with the corresponding mean R-R interval length. Multiple linear regression analysis examined the association between cardiac autonomic activity and indices of accumulated exercise stress.

Results: Significant inverse associations (95\% CI) between the Ln rMSSD:R-R ratio and both training load $[r=-0.48$ $(-0.70:-0.18), p=0.012]$ and time spent exercising above anaerobic threshold $[r=-0.51(-0.72:-0.22), p=0.006]$ were observed. The association between mean R-R interval length and Ln rMSSD was not significant $[r=0.05, p=0.950]$.

Conclusion: The current study demonstrated high intensity exercise, as defined through time spent above anaerobic threshold, displayed a stronger association with the Ln rMSSD:R-R ratio compared to a HR-derived training load when examining the dose response to accumulated intermittent exercise.

\section{Keywords: Heart rate variability - Cardiovascular physiology - Exercise physiology}

\section{Introduction}

Intermittent team sport involves frequent and repeated bouts of low and high intensity exercise that are largely reliant upon cardiorespiratory performance. Recent inquiry towards preferences of team sport practitioners for monitoring the dose-response to exercise has revealed significant reliance on the cardiovascular response to exercise during training and competition [25]. Team sports such as field hockey demand a high level of cardiorespiratory function in order to execute technical, tactical and physiological oriented activities throughout the entirety of a match [16]. The reliance on sustained cardiorespiratory performance has shown to elicit large heart rate (HR) derived training loads $[19,21]$ and prolonged periods of exercising above the anaerobic threshold [22]. This evidence supports the efficacy for integrative support staff within the sport of field hockey to concentrate on HR dynamics as part of their procedure for examining the dose-response to exercise.

Post exercise cardiac autonomic activity has demonstrated to be a valuable assessment for examining the doseresponse to exercise in intermittent team sport $[9,10]$. Alterations in cardiac autonomic activity can be examined through two distinct possibilities. The first, cardiac vagal tone, expressed through resting HR or the mean in successive R-R (ms) intervals, is a reflection of the action of the vagus nerve on the sinoatrial node, while the second, heart rate variability (HRV), conveys the magnitude of modulation between successive R-R intervals over a given recording period, and as such, one component may change without the other [13]. However, the analysis of HRV has been questioned when not accounting for vagal tone and its underlying influence on parasympathetic modulation $[5,11]$. 
The examination of each vagal index in relation to each other has been suggested to provide a more compelling assessment in cardiac automatic activity post endurance training [12]. Plews et al. established the efficacy for monitoring HRV in response to exercise stress in endurance athletes as a function of its corresponding R-R interval, whereby demonstrating the ratio between the two vagal indices may be more descriptive in displaying the doseresponse to exercise [24]. However, a polarized training model commonly utilized by endurance athletes fails to represent the intermittent nature of team sport, whereby HR dynamics can fluctuate in relation to both aerobic and anaerobic threshold. This difference between sports may be critical as exercise intensity is largely responsible for determining post-exercise HRV throughout a 48-hr period, where the suppression or supercompensation in vagal activity is dependent upon the exercise intensity experienced in relation to anaerobic threshold [27].

As such, this investigation examined the cardiac vagal modulation-to-vagal tone ratio and its association with accumulated exercise stress quantified through indices of cardiovascular strain; 1) a HR-derived training load and 2) time spent exercising above anaerobic threshold. It was hypothesized that time spent above anaerobic threshold would demonstrate a greater inverse association with the rMSSD:R-R ratio compared to a HR-derived training load.

\section{Material and methods}

\section{Participants}

Fourteen international calibre female field hockey players training as part of a national team were participants over a three-week period. Participant characteristics are provided in Table 1. All participants provided written informed consent to participate in the daily monitoring activities performed as members of the national team. The current investigation was approved by and followed the recommendations of the Clinical Research Ethics Board at the University of British Columbia and were in accordance with the ethical standards as laid down in the 1964 Declaration of Helsinki and its later amendments.

\section{Study design}

This investigation examined the association between accumulated exercise stress in intermittent team sport and the response in cardiac autonomic activity. Data collection took place during a three-week period where all participants trained together in a centralized location as members of the national team.

Training sessions were scheduled for Monday, Wednesday, and Friday of each week with Saturday and Sunday designated for complete rest. Training sessions were conducted in the morning and session start and finish times were standardized throughout the study. Weekly periodized exercise stress was decided by the national team's coaching staff and based on the objectives set forth towards developing the team's technical, tactical and physiological abilities. Training sessions followed a standardized structure beginning with a 15-min warm-up, followed by technical, tactical, and physiologically oriented field hockey drills, concluding with a 15-min cool down. Data collection and its analysis throughout the investigation was conducted by a standardized examiner.

\section{Heart rate derived training load}

Each participant was assigned a Polar ${ }^{\circledR}$ Team $^{2}$ heart rate monitor (Polar, Electro, Oy, Kempele, Finland) programmed to record at 1 -sec intervals. HR monitors were provided to each participant prior to beginning the warmup and were collected after completion of a cool down. Training loads were calculated from the start of the warmup until the completion of the cool-down automatically by the Polar ${ }^{\circledR}$ Team ${ }^{2}$ system and were developed utilizing the original training impulse method (TRIMP) $[3,4]$ that was customized with a recovery estimation model [17]. Each participant's maximum heart rate value used in the calculation of a training load was recorded from the graded maximal exercise test prior to entering the study, recent competition or during regular on-field training. The highest heart rate value observed was identified as the maximum heart rate and was utilized in the training load calculation model. Participants who did not complete a training session, or for whom any indices of exercise stress were not recorded over a single session, were excluded from analysis for the given week.

\section{Time spent exercising above anaerobic threshold ( $>$ LT2)}

Time (min) spent exercising above anaerobic threshold (>LT2) was determined using each participant's HR value recorded at anaerobic threshold and was automatically calculated by the Polar ${ }^{\circledR}$ Team ${ }^{2}$ system. Each

Table 1. Participant characteristics

\begin{tabular}{ccccc}
\hline Age $[\mathrm{y}]$ & Height $[\mathrm{cm}]$ & Weight $[\mathrm{kg}]$ & $\mathrm{BMI}\left[\mathrm{kg} / \mathrm{m}^{2}\right]$ & $\mathrm{VO}_{2 \mathrm{MAX}}\left[\mathrm{mL} \cdot \mathrm{kg}^{-1} \cdot \mathrm{min}^{-1}\right]$ \\
\hline $22.3 \pm 2.0$ & $168.7 \pm 5.3$ & $64.4 \pm 5.8$ & $22.6 \pm 1.4$ & $51.2 \pm 3.0$ \\
\hline
\end{tabular}


participant completed a graded maximal exercise test prior to entering the study. Participants were fitted with a mouthpiece consisting of a one-way non-rebreathing valve (Hans Rudolph, Kansas City, MO) for the collection of expired gas concentrations which were analysed using a calibrated metabolic cart (ParvoMedics TrueOne 2400 , Salt Lake City, UT). A treadmill grade of $2.0 \%$ was held throughout each test with a starting speed of 8.1 $\mathrm{km} / \mathrm{h}$. Each stage was $3 \mathrm{~min}$ in duration and involved increments of $0.8 \mathrm{~km} / \mathrm{h}$ until volitional fatigue. Alterations in expired gas concentrations were computed to identify ventilatory threshold / anaerobic threshold and its corresponding HR value.

\section{Cardiac parasympathetic activity}

Cardiac autonomic activity was examined through recording R-R intervals (ms) in the morning upon waking in the supine position [27] and were recorded each day over the weekend and were averaged to demonstrate parasympathetic response to accumulated exercise stress [20]. R-R intervals were recorded using a Polar ${ }^{\circledR}$ Team ${ }^{2}$ HR monitor equipped to sample at $1000 \mathrm{~Hz}$ and were exported to a computer software program, Kubios HRV 2.2 (Biosignal Analysis and Medical Imagining Group at the Department of Applied Physics, University of Kuopio, Kuopio, Finland) for analysis. A one-minute stabilization period [8] was utilized before using visual inspection for selecting a five minute artefact-free segment. [28]. Correction for ectopic beats or artefact that varied greater than $0.25 \mathrm{sec}$ when compared to the mean was automatically adjusted for with the use of a cubic spline interpolation method utilizing a third-degree polynomial pre-programmed into Kubios HRV 2.2 [1]. If one of two R-R recordings over the weekend was not collected or was invalid, that participant was not included in the weekly data set.

The square root of the mean squared differences of successive R-R intervals (rMSSD) was chosen for examining parasympathetic activity based on its efficacy to represent cardiac vagal modulation [28], its resilience against respiratory sinus arrhythmia during spontaneous breathing [18], and when not accounting for menstrual cycle phase [23]. Each rMSSD value was log-transformed (Ln rMSSD) using the natural logarithm to help reduce bias through possible non-uniformity in error. The relationship between vagal modulation and vagal tone was demonstrated as a ratio and was calculated using Ln rMSSD and its corresponding R-R (ms) interval (Ln rMSSD:R-R). Resting heart rate was determined from the same five-minute $\mathrm{R}-\mathrm{R}$ recording period and was calculated in conjunction with HRV analysis using $\mathrm{Ku}-$ bios HRV 2.2.

\section{Statistical analysis}

All data in text, figures, and tables are displayed as a mean $( \pm \mathrm{SD})$ unless otherwise stated. A repeated measures design was utilized to examine variation in each index of exercise stress and parasympathetic activity using a one-way, repeated measures ANOVA with a Bonferroni correction procedure for post-hoc comparisons. Significance was declared using a probability of $p<0.05$. A Shapiro-Wilk's test of normality $(p>0.05)$ was conducted and revealed a range in Kurtosis of $(-0.57: 0.28)$ and a range in Skewness of $(-0.03: 0.46)$.

Multiple linear regression analysis $\left(\mathrm{R}^{2}\right)$ was employed using $\mathrm{VO}_{2 \mathrm{MAX}}$ as a covariate for its influence on post-exercise cardiac autonomic activity [6] to examine the association between the Ln rMSSD:R-R ratio and: 1) a HR derived training load and, 2) time spent exercising above anaerobic threshold. Additionally, a Pearson Correlation coefficient $(r)$ and its corresponding 95\% confidence interval was conducted separately to examine individual associations between each index of exercise stress and each index of cardiac autonomic activity. The following principles were applied for identifying the strength of correlation; $0.10-0.29$ small, $0.30-0.49$ moderate, $>0.50$ large [7].

Table 2. Multiple linear regression analysis examining parasympathetic activity and accumulated exercise stress

\begin{tabular}{|c|c|c|c|c|c|c|}
\hline Models & Standard error & $t$ & $F$ & $r(95 \% \mathrm{CI})$ & $\mathrm{R}^{2}$ & $p$-value \\
\hline Ln rMSSD : R-R (ms) & 0.000 & & 5.99 & $-0.51(-0.72:-0.22)$ & 0.26 & 0.006 \\
\hline Time (min) Above Threshold & 0.000 & 2.03 & & & & 0.050 \\
\hline $\mathrm{VO}_{2 \mathrm{MAX}}$ & 0.000 & -1.44 & & & & 0.158 \\
\hline Ln rMSSD : R-R (ms) & 0.000 & & 5.09 & $-0.48(-0.70:-0.18)$ & 0.23 & 0.012 \\
\hline Training Load (AU) & 0.000 & 1.62 & & & & 0.114 \\
\hline $\mathrm{VO}_{2 \mathrm{MAX}}$ & 0.000 & -2.53 & & & & 0.016 \\
\hline Ln rMSSD (ms) & 0.469 & & 0.05 & $0.05(-0.28: 0.37)$ & 0.003 & 0.953 \\
\hline $\mathrm{R}-\mathrm{R}(\mathrm{ms})$ & 0.001 & 0.31 & & & & 0.761 \\
\hline $\mathrm{VO}_{2 \mathrm{MAX}}$ & 0.026 & 0.06 & & & & 0.954 \\
\hline
\end{tabular}




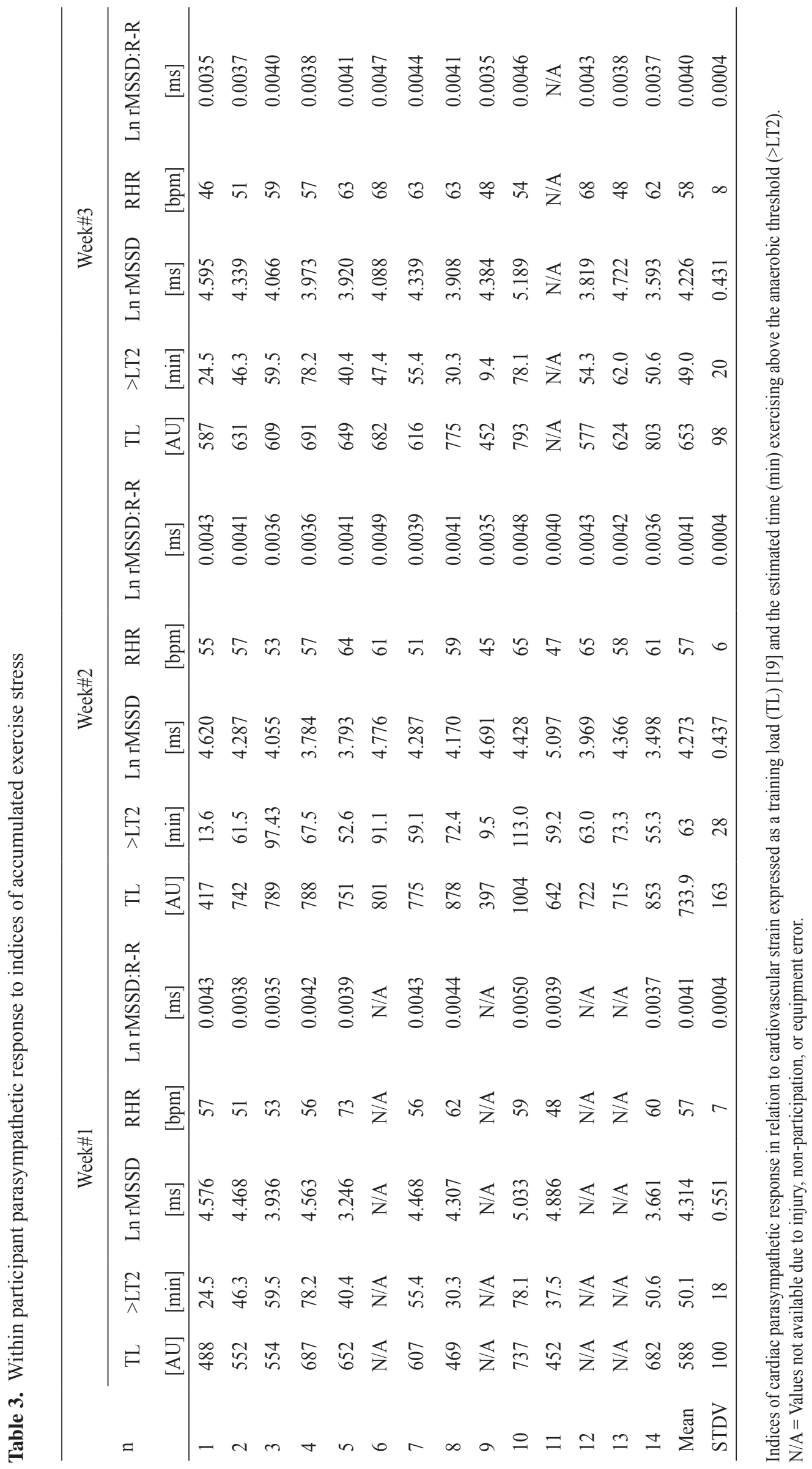




\section{Results}

A total of 37 data sets out of a possible 42 were analysed. Missing data sets were the result of HR monitors failing to record either R-R intervals or a HR-derived training load value in one of three exercise sessions within the week. Significant inverse associations between the Ln rMSSD:R-R ratio and both HR-derived training loads $[r=-0.48(-0.70:-0.18), p=0.012]$ and time spent above anaerobic threshold $[r=-0.51(-0.72:-0.22), p=0.006]$ were observed (Table 2). Within participant variation in indices of cardiovascular strain and parasympathetic activity remained unremarkable over the three-week investigation (Table 3 ). The association between mean R-R interval length and Ln rMSSD was not significant $\left[\mathrm{R}^{2}=0.003\right.$, $p=0.950]$. Individual associations between indices of exercise stress and indices of cardiac autonomic activity are presented in Figure 1.

\section{Discussion}

This investigation examined indices of accumulated intermittent exercise stress when quantified through cardiovascular strain and their association with the Ln rMSSD:R-R ratio over a three week period. The main findings of this study were as follows; 1) each index of exercise stress demonstrated significant and inverse associations with the HRV to vagal tone ratio, and 2) change in the ratio was the result of alterations in both vagal tone and vagal modulation.

The comparable strength of association observed between the Ln rMSSD:RR ratio and each index of exercise stress may be the consequence of cardiac autonomic response after performing different levels of exercise intensity. HR-derived training loads typically quantify exercise HR dynamics in relation to both aerobic and anaerobic threshold. When exercising above the anaerobic threshold, an immediate 24-hr suppression in HRV is observed, that eventually supercompensates above baseline values at 48-hr post-exercise [27]. This undulating response is in contrast to the immediate 24-hr elevation in HRV when exercising at the aerobic threshold, which eventually diminishes below baseline levels at 48-hr post exercise [27]. The current study examined cardiac autonomic activity over a 48-hr period and may have allowed for alternating dynamics in HRV to produce comparable associations between each index of exercise stress and the Ln rMSSD:RR ratio. These findings are in support of previous literature whereby demonstrating a similar strength of association between day-to-day variation in Ln rMSSD (ie. Ln rMSSD ${ }_{\mathrm{CV}}$ ) over a 48-hr period and both a HR-derived training load and time spent exercising above anaerobic [20]. Collectively, these results support the utility of global and high intensity indices of a)

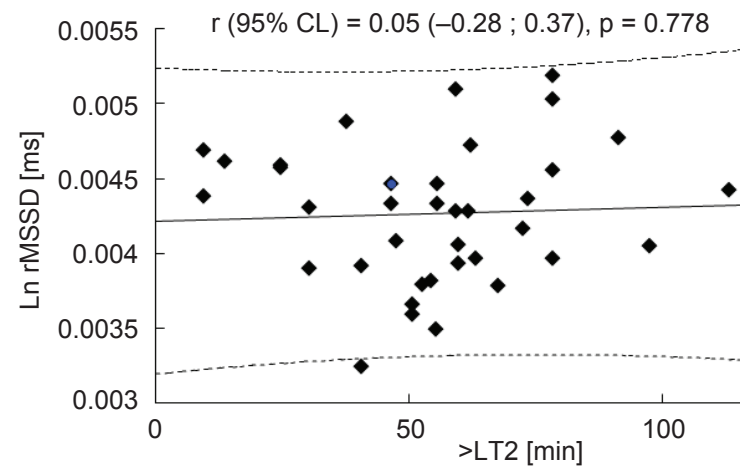

c)

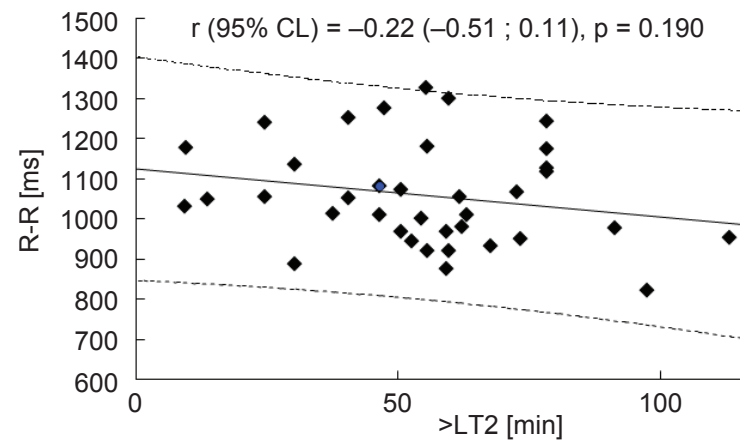

b)

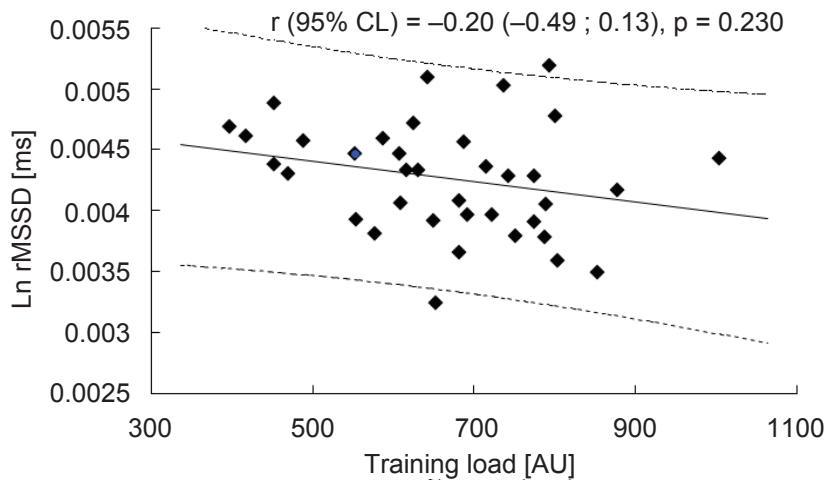

d)

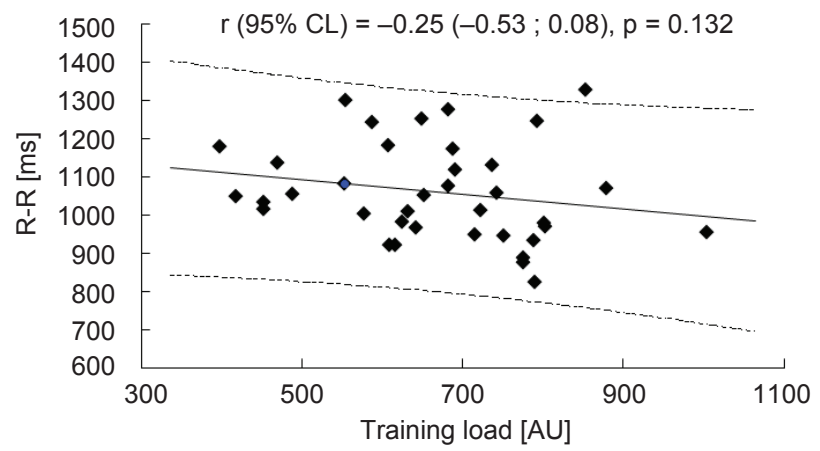

Fig. 1. Association between each index of exercise stress and indices of cardiac parasympathetic activity 
cardiovascular strain when examining the dose response to exercise using cardiac autonomic activity in team sport athletes.

Post exercise, vagal indices of HRV have shown to be a valuable tool in assessing an athlete's physiological response to exercise [2]. However, the sole reliance on examining HRV may not be sufficient for interpreting an athlete's response to exercise. Similar HRV values have been observed in elite endurance athletes during periods of both normal training and non-functional overreaching, a result that can be displayed through a bell-shaped curve in HRV in relation to R-R interval length [24]. Differences in cardiac autonomic response between intermittent team sport and endurance sport may be largely determined through their distinct training methods. Endurance athletes typically utilize a polarized training model that focuses on performing the majority of exercise with an intensity at the aerobic threshold, with the remainder of training performed above anaerobic threshold [26]. Constant and prolonged exercise at aerobic threshold has shown to develop the saturation of acetylcholine receptors at the sinoatrial node, whereby eliciting heightened vagal tone and sustained parasympathetic dominance, with concomitant reductions in respiratory heart sinus arrythmia HRV [15]. The current study demonstrated change in the Ln rMSSD:R-R ratio as a result of alterations in both vagal modulation and vagal tone. Although the respective association between each index of exercise stress and both HRV and vagal tone was not significant, our results identified unique tendencies in vagal activity in response to accumulated exercise stress (Fig. 1). HRderived training loads demonstrated an inverse association with both $\operatorname{Ln} \operatorname{rMSSD}(r=-0.23, p=0.230)$ and R-R interval length $(r=-0.25, p=0.132)$. In contrast, the association between time spent above anaerobic threshold and Ln rMSSD demonstrated an almost non-existent relationship ( $r=0.05, p=0.778)$ while vagal tone demonstrated an inverse association $(r=-0.22, p=0.190)$ This difference elicited a larger ratio and a stronger relationship between time spent above threshold and the Ln rMSSD:R-R ratio (Table 2).

The current investigation is limited to examining exercise stress in the form of on-field training. It remains undetermined where the strength of associations may have been altered with the inclusion of resistance training. As such, we must acknowledge the influence of resistance training on daily parasympathetic HRV indices [14] and its potential impact on altering the strength of association between total weekly accumulated exercise stress and the HRV to vagal tone ratio. We encourage future studies to include concurrent training styles in intermittent team sport to expand on our findings.

\section{Conclusion}

In summary, cardiovascular strain when expressed as a HR-derived training load or time spent exercising above anaerobic threshold displayed a significant inverse association with the Ln rMSSD to R-R ratio. Alterations in the ratio were the result of variation in both vagal tone and parasympathetic modulation. Although time spent above threshold demonstrated a marginally stronger association, our results suggest practitioners may concentrate on either index of cardiovascular strain when examining the dose response to intermittent exercise using the Ln rMSSD:R-R ratio. Preference may be given towards indices that are analysed in a time efficient method, allowing rapid evidencebased decision making between coach and practitioner.

Conflict of interest: Authors state no conflict of interest.

\section{References}

1. Alcantara J., Plaza-Florido A., Amaro-Gahete F.J., Acosta F.M., Migueles J.H., Molina-Garcia P., Sacha J., Sanchez-Delgado G., Martinez-Tellez B. (2020) Impact of Using Different Levels of Threshold-Based Artefact Correction on the Quantification of Heart Rate Variability in Three Independent Human Cohorts. J. Clin. Med., 9: 325.

2. Aubert A.E., Seps B., Beckers F. (2003) Heart rate variability in athletes. Sports Med., (Auckland, NZ) 33: 889-919.

3. Banister E.W. (1991) Modeling Elithe Athletic Performance In: MacDougall J.D., Wenger H.A., Green H.J. (eds.) Physiological Testing of Elite Athletes. Champaign, Illinois: Human Kinetics.

4. Banister E.W., Morton R.H., Fitz-Clarke J. (1992) Dose/ response effects of exercise modeled from training: physical and biochemical measures. Ann. Physiol. Anthropol., 11: 345-356.

5. Bolea J., Pueyo E., Orini M., Bailon R. (2016) Influence of Heart Rate in Non-linear HRV Indices as a Sampling Rate Effect Evaluated on Supine and Standing. Frontiers in Physiology, 7: 501.

6. Buchheit M., Mendez-Villanueva A., Quod M.J., Poulos N., Bourdon P. (2010) Determinants of the variability of heart rate measures during a competitive period in young soccer players. Eur. J. Appl. Physiol., 109: 869-878.

7. Cohen J. (1988) Statistical Power Analysis for the Behavioral Sciences. Hillsdale, NJ: Lawrence Erlbaum Associates Inc. 
8. Flatt A.A., Esco M.R. (2016) Heart rate variability stabilization in athletes: towards more convenient data acquisition. Clin. Physiol. Funct. Imaging, 36: 331-336.

9. Flatt A.A., Esco M.R., Nakamura F.Y. (2017) Individual Heart Rate Variability Responses to Preseason Training in High Level Female Soccer Players. J. Strength Cond. Res., 31: 531-538.

10. Flatt A.A., Esco M.R., Nakamura F.Y., Plews D.J. (2017) Interpreting daily heart rate variability changes in collegiate female soccer players. J. Sports Med. Phys. Fit., 57: 907-915.

11. Goldberger J.J,. Ahmed M.W., Parker M.A., Kadish A.H. (1994) Dissociation of heart rate variability from parasympathetic tone. Am. J. Physiol.-Heart Circ. Physiol., 266: H2152-H2157.

12. Goldberger J.J., Challapalli S., Tung R., Parker M.A., Kadish A.H. (2001) Relationship of heart rate variability to parasympathetic effect. Circulation 103: 1977-1983.

13. Hedman A., Hartikainen J., Tahvanainen K., Hakumäki M. (1995) The high frequency component of heart rate variability reflects cardiac parasympathetic modulation rather than parasympathetic 'tone'. Acta Physiologica Scandinavica, 155: 267-273.

14. Kingsley J.D., Figueroa A. (2016) Acute and training effects of resistance exercise on heart rate variability. Clin. Physiol. Funct. Imaging 36: 179-187.

15. Malik M., Camm A.J. (1993) Components of heart rate variability - what they really mean and what we really measure. Am. J. Cardiol., 72: 821-822.

16. McGuinness A., Malone S., Petrakos G., Collins K. (2017) The Physical and Physiological Demands of Elite International Female Field Hockey Players During Competitive Match-Play. J. Strength Cond. Res., 33(11): 3105-3113.

17. Nissilä J., Kinnunen H. (2008) Heart rate based training load and recovery time estimation. Polar Electro Oy 1: $1-4$.

18. Penttilä J., Helminen A., Jartti T., Kuusela T., Huikuri H.V., Tulppo M.P., Coffeng R., Scheinin H. (2001) Time domain, geometrical and frequency domain analysis of cardiac vagal outflow: effects of various respiratory patterns. Clin. Physiol., 21: 365-376.

19. Perrotta A.S., Held N.J., Warburton D.E.R. (2017) Examination of internal training load parameters during the selection, preparation and competition phases of a mesocycle in elite field hockey players. Int. J. Perf. Anal. Sport, 17: 813-821.

20. Perrotta A.S., Koehle M.S., White M.D., Taunton J.E., Warburton D.E.R. (2019) Consecutive non-training days over a weekend for assessing cardiac parasympathetic variation in response to accumulated exercise stress. Eur. J. Sport Sci., 1-11.
21. Perrotta A.S., Taunton J.E., Koehle M.S., White M.D., Warburton D.E.R. (2018) Monitoring the Prescribed and Experienced Heart Rate Derived Training Loads in Elite Field Hockey Players. J. Strength Cond. Res., 33(5): 1394-1399.

22. Perrotta A.S., Warburton D.E.R. (2018) A comparison of sessional ratings of perceived exertion to cardiovascular indices of exercise intensity during competition in elite field hockey players. Biomed. Hum. Kinet., 10: 157-162.

23. Pestana E.R., Mostarda C.T., Silva-Filho A.C., Salvador E.P., de Carvalho W.R.G. (2018) Effect of different phases of menstrual cycle in heart rate variability of physically active women. Sport Sci. Health, 14: 297-303.

24. Plews D.J., Laursen P.B., Kilding A.E., Buchheit M. (2012) Heart rate variability in elite triathletes, is variation in variability the key to effective training? A case comparison. Eur. J. Appl. Physiol., 112: 3729-3741.

25. Schneider C., Hanakam F., Wiewelhove T., Doweling A., Kellmann M., Meyer T., Pfeiffer M., Ferrauti A. (2018) Heart Rate Monitoring in Team Sports-A Conceptual Framework for Contextualizing Heart Rate Measures for Training and Recovery Prescription. Frontiers in Physiology, 9: 639.

26. Seiler K.S., Kjerland G.O. (2006) Quantifying training intensity distribution in elite endurance athletes: is there evidence for an "optimal" distribution? Scand. J. Med. Sci. Sports, 16: 49-56.

27. Stanley J., Peake J.M., Buchheit M. (2013) Cardiac parasympathetic reactivation following exercise: implications for training prescription. Sports Med., 43: 1259-1277.

28. Variability H.R. (1996) Standards of measurement, physiological interpretation, and clinical use. Task Force of the European Society of Cardiology and the North American Society of Pacing and Electrophysiology. Circulation 93: 1043-1065.

\section{Received 28.05.2020 \\ Accepted 18.06.2020}

\section{(C) University of Physical Education, Warsaw, Poland}

\section{Acknowledgments}

The authors would like to acknowledge the support, dedication, and contributions from each participating athlete. The results of the current study do not constitute endorsement of the products utilized by the authors or the journal of Biomedical Human Kinetics. This investigation was funded through the Natural Sciences and Engineering Research Council of Canada (RGPIN-2018-04613). 